\title{
The Mass Loss Rate - Period Relation in Carbon Miras
}

\author{
Martin Groenewegen \\ Institut d'Astrophysique de Paris, CNRS, 98 bis Boulevard Arago, \\ F-75014 Paris, France
}

\begin{abstract}
The relation between mass loss rate and pulsation period in carbon Miras is discussed. The dust mass loss rate is very low (about $210^{-10} \mathrm{M}_{\odot} / \mathrm{yr}$ ) up to about $P=380$ days, where there is a sudden increase. For $P>400$ days there is a linear relation between $\log \dot{M}$ and $P$. The change in the mass loss rate near 380 days may be related to radiation pressure on dust becoming effective in driving the outflow.
\end{abstract}

\section{Introduction}

The mass loss rate is the determining factor in the evolution of Asymptotic Giant Branch (AGB) stars since the time scale on which mass loss takes place is usually much shorter than the time scale of the nuclear processes.

As on the other hand it is well known that most AGB stars are variable and that it is believed that mass loss and pulsation are connected it is useful to study the relation between mass loss and pulsation period. The $\dot{M}-P$ relation has been studied in quite some detail for oxygen-rich Miras (Schild 1989, Wood 1990, Whitelock 1990, Whitelock et al. 1994) but never for carbon-rich Miras. In this contribution such a relation is presented (also see Groenewegen 1995, where this relation is compared to the ones derived for oxygen-rich Miras).

\section{Results}

Thirty-two carbon Miras were selected from the General Catalog of Variable Stars, Jones et al. (1990) and Le Bertre (1992). The spectral energy distributions and IRAS LRS spectra were fitted using the dust radiative transfer model of Groenewegen (1993).

The relation between the dust mass loss rate and pulsation period is shown in Fig. 1 (top panel). Two features stand out: there is a tight correlation above 400 days, and there appears to be a significant increase in the dust mass loss rate near $P=\mathbf{3 8 0}$ days.

A different tracer of the dust formation process is the dust-to-gas (DTG) ratio. In the bottom panel the DTG ratio is plotted versus $P$. The gas mass loss rates as derived from CO measurements were taken from Loup et al. (1993) and scaled to the adopted distances. The DTG ratio increases with period for $P \gtrsim 400$ days, suggesting a more efficient dust production. 

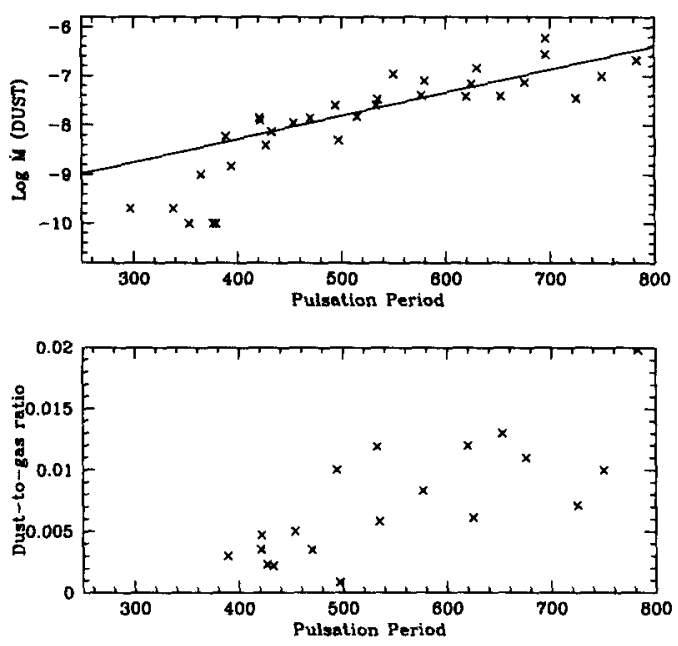

Figure 1. The dust mass loss rate and dust-to-gas ratio in the sample of carbon Miras. The solid line is a least-squares fit for $P>400$ days.

As is evident from Fig. 1 there is very little data available for shorter periods. I am engaged to obtain near-infrared photometry, mid-infrared spectra and $\mathrm{CO}$ data for more short period carbon Miras.

Recently, Netzer \& Elitzur (1993) and Habing et al. (1994) predicted a lower limit of about $10^{-7} \mathrm{M}_{\odot} / \mathrm{yr}$ to the (total) mass loss rate to sustain an outflow driven by radiation pressure on dust. With $\Psi=0.003$ near $P=380$ days, a total mass loss rate of $810^{-8} \mathrm{M}_{\odot} / \mathrm{yr}$ is derived, close to this lower limit. Thus, the increase in the dust mass loss rate near 380 days may be related to radiation pressure on dust becoming effective and hence provide observational evidence for the theoretical prediction of Netzer \& Elitzur and Habing et al.

\section{References}

Groenewegen, M.A.T. 1993, Ph.D. thesis, University of Amsterdam Groenewegen, M.A.T. 1995, in: Circumstellar Matter, ed. G. Watt, in press Habing, H.J., Tignon, J., \& Tielens, A.G.G.M. 1994, A\&A, 286, 523 Jones, T.J., et al., 1990, ApJS, 74, 785

Le Bertre, T. 1992, A\&AS, 94, 377 Loup, C., Forveille, T., Omont, A., \& Paul, J.F. 1993, A\&AS, 99, 231

Netzer, N., \& Elitzur M. 1993, ApJ, 410, 701

Schild, H. 1989, MNRAS, 240, 63

Whitelock, P. 1990, ASP Conf. Ser., 11, 365

Whitelock, P., et al. 1994, MNRAS, 267, 711

Wood, P.R. 1990, in: From Miras to Planetary Nebulae, Editions Frontieres: Gif-sur-Yvette, p. 67 